\title{
La Ingenierizacion y la Ingenieria Concurrente em los Proyectos de la Industria Farmaceutica y la Biotecnologia
}

\author{
Silvio Viña Brito
}

Doctor en Ciencias Técnicas, Inginiero Industrial, Profesor Titular, Facultad de Ingenieria Industria del Instituto Superiore Politécnico "José A. Echeverria"

\section{Eduardo Concepción Batiz}

\section{Ricardo Montero Martinez}

Ingeniero Industrial, Profesor Asistente, Facultad de Ingenieria Industrial del Instituto Superior Politécnico "José A. Echeverria"

Palabras claves: escalado, ingenieria concurrente ó simultánea, industria biotecnológica, industria farmacéutica, diseño

Key words: scaling-up, concurrent or simultaneus engineering, biotechnological industry, pharmaceutical industry, design.

\section{RESUMEN}

El proceso de escalado en la industria Farmaceútica y Biotecnológica puede presentar problemas si no se cumplen determinados requesitos. Se ha supuesto que la llamada ingenierización puede contribuir a reducir esos problemas. Se analizan conceptos como el ciclo del producto, el escalado y su relación con la ingenierización. Se discutc el concepto de ingenieria concurrente o simultânea y se detallan aspectos que deben tenerse en cuenta para hacer más efectivo el proceso.de ingenierización y el escalado.

\section{ABSTRACT}

The scaling-up process in the Pharmaceutical and Biotechnological industry may shown problems if several requeriments are not accomplished. It has been thought that the so called "ingineerization" can contribute to reduce those problems. Several concepts such as product cicle scaling-up and their relation with ingineerization" are analyzed. The concept of concurrent or simultaneous engineering is introduced and some aspects to bear in mind for increasing the efficiency the efficiency of this process are also analyzed. 


\section{Introducción}

Tanto en el Primer Taller Internacional de Escalado como en el Segundo, desarrollados en Cuba en 1992 y 1993, auspiciados por el Centro Químico Farmacéutico (CQF) y que sesionaron en el Centro de Ingenieria Genética y Biotecnologia (CIBG), fueron analizados los problemas que podrian encontrase al tratar de aumentar la producción en las ramas Biotecnológica y Farmacéutica hasta un nivel industrial.

Por ejemplo, a veces las reacciones y procesos empleados a nivel de laboratorio no alcazaban el mismo rendimiento cuando se aumentaba el volumen (o se trataba de aumentar la capacidad de producción).

Supóngase el caso de un alimento animal cuyo contenido de proteína en el proceso industrial no logra siempre ser tan alto como en los experimentos de laboratorio. Este caso podria ser por ejemplo principalmente originado a partir de un problema en el escalado de una fermentación pero conviene alertar que otros factores tales como el envase, la manipulación y el transporte pueden complicarse al aumentar la escala de producción.

Se pudieran citar otros casos donde logros e conocimientos cientificos fundamentados a pequeña escala no han podido generalizarse o escalarse porque otros factores no lo han permitido.

El presente artículo tiene como objetivos analizar los problemas y los procedimientos que se han empleados en otras áreas, evidenciar su similitud con la industria Biotecnológica y Farmacéutica, asi como brindar un conjunto de recomendaciones que podrían tenerse en cuenta para mejorar la eficiencia de los proyectos en esta rama.

\section{Relación entre}

\section{ingenierización, escalado y el ciclo de vida del producto}

En los citados eventos, los cientificos participantes (principalmente licenciados químicos, biólogos, farmaceúticos) honestamente reconocían que algunos resultados reportados se resistian a su producción industrial y que algunos casos demoraban mucho tiempo en el proceso de escalado. Alertaban que eran necesarios los ingenieros y se recomendó dar pasos para lograr lo que se denominóla ingenierización de los procesos químicos y bioquímicos. Este concepto de ingenierización es limitado, lo cual se verá posteriormente.

El ciclo de vida de un producto es el tiempo que transcurre desde su concepción hasta su comercialización. Pudiera incluir el tiempo de uso o consumo hasta que se deseche y se recupera e incluir las actividades de mantenimiento, servicio y garantia (pudiera establecerse una diferencia entre el ciclo de producción y el ciclo de vida).

Se define como escalado: el proceso necesario para alcanzar la producción industrial a partir de un logro cientifico a nivel del laboratorio" (ICDCA, 1992). El escalado de procesos químicos y biotecnológicos generalmente es realizado según las siguientes etapas: concepción, laboratorio, banco, planta piloto, producción industrial y comercialización. Etapas similares se pueden encontrar en la manufactura: concepción, maqueta, prototipo, serie cero, producción industrial y comercialización.

Al analizar estas definiciones se hacen 
evidentes las relaciones entre los conceptos. Los eventos, las actividades, el tiempo que transcurre, es el ciclo de vida. El proceso que une, que genera estos eventos durante el ciclo de vida, o de producción, es el escalado. A la participación de ingenieros $\mathrm{y}$ al uso de las técnicas de ingenieria en esos procesos se ha denominado ingenierización.

Por qué es importante el concepto de ciclo de vida?

Se plantea con insistencia que la ventaja que ha estado mostrando la industria japonesa sobre la norteamericana y la europea se debe, entre otros factores, a que el ciclo de procucción de las empresas japonesas es mucho menor (Morita, 1992). Desde la óptica competitiva industrial, este ciclo de producción que es parte del ciclo de vida, tiene una importancia vital. Algunos ejemplos:

- la industria automovilistica casi la inventaron en los Estados Unidos (FORD), pero el desarrollo de un nuevo modelo de carro hoy en dia, demora en los Estados Unidos 62 meses y en Japón 43 (Morita, 1992);

- en la electrónica de consumo el que demora mas de un año desde que concibe el producto hasta que lo comercializa, no compite (Europace System, 1992).

\section{Por qué es ventajoso el ciclo más corto?}

Vamos a suponer que se detecta una cierta necesidad de un producto (el mercado, la ventana, el nicho: una demanda no satisfecha). Es probable que varias entidades en todo el mundo se enteran, por ejemplo, en el campo de la biotecnologia, deben estar trabajando en la obtención de la vacuna del SIDA cientos de instituciones. El que llega primero al mercado impone el precio (con muchas ganancias). Cuando los productores son po$\cos$, aún se mantiene el precio, pero eventualmente comienza a bajar, si aumenta la oferta. En el gráfico 1 se representa esta idea. Entonces quienes tengan el ciclo mas corto, tendrán mayores posibilidades de mantenerse en la competencia.

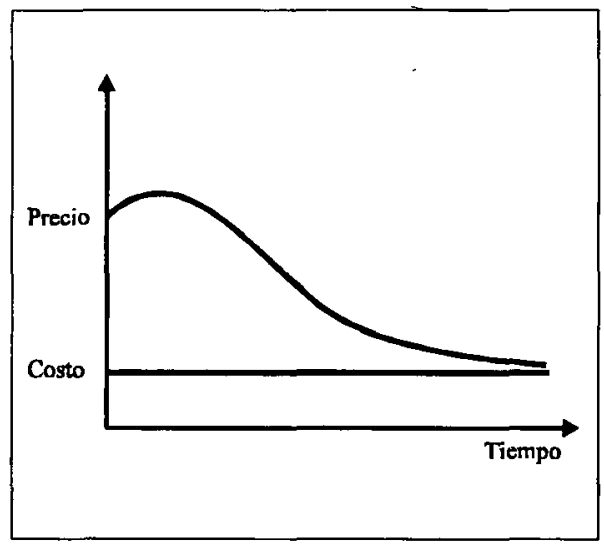

Figura 1

Variación del Precio del Producto em el Tiempo

El acortamiento del ciclo influye en otros aspectos que pueden ejemplificarse en los dos siguientes: los inventarios disminuyen (en las industrias referidas este es un efecto importante teniendo en cuenta que muchos productos tienen vida limitada), y los costos fijos influyen durante menos tiempo.

\section{Cuáles son los factores que influyen en el alargamiento del ciclo?}

Recordemos las etapas: concepción, laboratorio, banco, planta piloto, producción industrial y comércialización. Generalmente estas etapas o pasos se realizan en secuencia e incluso por distintas personas. Se describirá con mayor detalle lo que se incluye en las etapas, con posibles variaciones.

En el diseño conceptual y de laboratorio se 
realiza la investigación de mercado, la investigación de laboratorio, obtención de pequeñas cantidades para pruebas de efectos biológicos, prueba con animales de laboratorio y determinación de métodos de obtención y evaluación (metrologia, etc) y comprobación de la factibilidad.

En el trabajo de banco se hace un escalado de 1:3 - 1:5, se afinan los métodos, se trata de aumentar la eficiencia, se obtienen mayores cantidades del producto para la realización de pruebas en animales o clinicas con mayor masividad, frecuentemente se comienzan las tareas para el registro del producto y se revisan los estudios de factibilidad.

Hasta este momento, y según las experiencias de los autores, habiendo transcurrido aproxima damente el $20 \%$ del tiempo del ciclo de producción del producto, tipicamente se han tomado alrededor del $80 \%$ de las decisiones sobre el diseño del producto en si y sobre la tecnologia, las cuales determinan igualmente alrededor del $90 \%$ de los costos, sin haber intervenido en general los expertos de fabricación, aseguramiento y marketing, entre otros.

En la planta piloto se hace otro escalado, se determinan en detalle los consumos de materiales y energia, se precisan los rendimientos, debiendo comprobarse las hipótesis y la factibilidad técnica y económica.

Si los ingenieros y otros expertos no han participado integramente desde el inicio de la investigación se puede perder mucho tiempo en esta etapa. Por ejemplo: con frecuencia, falta información para el diseño del producto y de las plantas de producción que hubiera sido fácil determinar en las primeras etapas, pero que no son interesantes para los investigadores, sino para los diseñadores de la planta piloto y de producción (densidad, viscosidad, temperaturas críticas, metrologia para el aseguramiento de la calidad, tiempos en general de operaciones principales y auxiliares, requisitos de la materia prima, - puesto que se ha venido trabajando con reactivos -, requisitos del aseguramiento y del envase).

En la Producción se proyecta la planta y se ejecuta su construcción y montaje (lo cual puede demorar años), así como se prepara el personal de producción.

En esta etapa se pueden presentar muchos problemas heredados de la falta de integralidad en el análisis durante las etapas anteriores. Puede suceder que se comience a proyectar e incluso a construir, suponiéndose lo que la planta debia costar. Según avanza el proyecto y la construcción, van "apareciendo" elementos que elevan los gastos, así como decisiones forzadas en parte por lo ya invertido, que conducen a un costo total superior, que de haberse conocido inicialmente, hubiera tenido alguna probabilidad de no haberse aprobado.

Entre tales problemas pueden estar:

- Contaminación ambiental: El escalado puede aumentar la contaminación a niveles no admisibles (valores superiores a los limites de recuperación del medio). Un efecto relacionado con la contaminación es el riesgo de incedio, explosión y otros que pueden tener características similares.

- Recursos para el envasado no considerados: A veces el envase tiene un costo mayor que el producto envasado.

- Servicios auxiliares que se han dado por existentes: energia eléctrica, vapor, tratamiento de desechos, aire acondicionado y limpi- 
eza del aire.

Almacenamiento en condiciones especiales: Necesidad de que algunos productos se mantengan en atmósferas controladas, requiriendo por ejemplo temperaturas y humedades relativas bajas con una alta fiabilidad, lo que adicionalmente presupone la necesidad de la instalación de equipamiento de reserva para casos de fallos de los que están en operación.

- Ubicación y conservación de productos en proceso: De no tenerse en cuenta desde un inicio la necesidad del almacenamiento de los productos en proceso, puede conllevar al abarrotamiento, ó incluso a la imposibilidad de alcanzar las producciones proyectadas.

- Aseguramiento en general: Las pequeñas cantidades de productos necesarios para el trabajo en laboratorio, pueden obterner-se en ocasiones por relaciones informales y $\sin$ mediar relaciones econômicas mercantiles. Al pasar a la producción industrial debe preverse que son necesarios volúmenes mucho mayores que no pueden obternerse por esa via.

En la comercialización debe tenerse en cuenta las redes de ventas, la propaganda, el mantenimento y los servicios de garantia.

Se puede concluir que cualquiera de estos factores puede impedir el éxito de las comercialización del producto generado por el logro e descubrimiento científico.

\section{Cual es la solución?}

Un concepto utilizado muy frecuentemente en la actualidad y que puede contribuir a la solución de los problemas anteriormente senãlados es el de Ingenieria simultánea o con- currente (diseño concurrente, o a veces Team approach), cuya definición es: “... un enfoque sistemático al diseño integrado, concurrente, de los productos y los procesos relacionados con ellos, incluyendo su fabricación y aseguramiento. La intención de este enfoque es obligar a los que desarrollan el producto, desde el principio a considerar todos los elementos del ciclo de vida del producto, desde su concepción hasta su distribución incluyendo los requisitos de calidad, costo, programación y uso". (Rhyne, 1991: Europace System, 1992; Viña, 1990; Xerox, 1986).

La idea de la ingenieria concurrente no es nueva. Existen ejemplos en que un enfoque similar funcionó, en ocasiones por características excepcionalmente integrales del promotor, tal es el caso de Ford en la industria automovilística.

\section{Como se realiza en la práctica?}

Conformación de un grupo multidisciplinario de expertos, desde el mismo comienzo del proyecto (el título no es lo más importante, un experto puede ser originalmente graduado de ingenieria idnustrial, química ó mecánica, entre otros especialistas), pudiéndose tentativamente incluir:

1. expertos en fabricación, manufactura;

2. expertos en aseguramiento (compras, adquisición de materiales y dispositivos);

3. expertos en conservación del medio ambiente (tratamiento de residuales, reciclaje);

4. expertos en eliminación de la contaminación y control del microclima;

5. expertos de envases, embalajes y mani- 
pulación de materiales y su conservación;

6. expertos en aseguramiento de la calidad;

7. expertos en energética;

8. expertos en recursos humanos (condiciones laborales, diseño de puestos de trabajo, métodos, entrenamiento, seguridad industrial);

9. otros expertos según el caso.

Por la lista anterior, se puede apreciar que los integrantes del grupo no deben ser sólo ingenieros, por lo cual a nuestro criterio el concepto de ingenierización antes señalado es aún estrecho para cubrir las necessidades descritas.

La eliminación del proceso de diseño secuencial potencialmente elimina o reduce los problemas que pudieran aparecer después, algunos de los cuáles han sido esbozados anteriormente.

Estos grupos de expertos deben conformarse con determinados requisitos:

- uso de técnicas de trabajo en grupo y otras de solución de problemas (Xerox, 1986);

- facilidades de comunicación;

· base de datos común accesible;

- local de trabajo, servicios, de oficina y generales.

Algunos obstáculos que pueden presentarse para la implementación de la ingenieria simultánea ó concurrente son: (Ziemke y Spann, 1991):
- departamentalización estructural;

- sistemas de estimulación diseñados para el individuo;

- dirigentes apegados a dar soluciones simples a problemas complejos.

Las técnicas de la ingenieria concurrente deben contribuir a que se tengan en cuenta cuanto antes, aspectos frecuentemente olvidados. A continuación, según una clasificación convencional y sin pretender un orden ni agotar el tema, se citan algunos:

\section{Producibilidad (Salvendy, 1982)}

- factibilidad de los procesos a escala industrial y sus alternativas tecnológicas;

- información requerida para el diseño de plantas en el caso de la biotecnologia, por ejemplo: patogenicidad, viabilidad, nivel de inventarios, procedimientos de limpieza y manipulación (Whalen, 1991);

- factibilidad económica. Producción minima. Balance en divisas;

- demanda y su pronóstico;

- envase y conservación de los productos en proceso y terminados;

- requisitos de automatización y alternativas de solución;

- buenas prácticas de fabricación y de mantenimiento;

- aseguramiento de la calidad (metrologia, muestreos);

- balance energético, requisitos de emer- 
gencia;

- programación de la producción (también su relación con el régimen de trabajo y descanso);

- requisitos ambientales. Limpieza del aire. Efectos sobre los esquemas de circulación. Aspectos econômicos y de mantenimiento. Selección de la clase de área limpia. Sistemas de limpieza del aire y de acondicionamiento del aire. Presiones en los locales. Compuertas. Filtros HEPA (High Efficiency Particulate Air) y flujos laminares (Federal Standard, 1990; ICIDCA, 1992; Patel and Greiner, 1991);

- grado de contención;

- desechos y su posibilidad de neutralización (por ejemplo la recuperación de solventes).

\section{A seguramiento}

- disponibilidad de materias primas. Requisitos de calidad. Plazos de entrega;

- disponibilidad de otros materiales, combustibles y energía en general;

- disponibilidad de envases;

- almacenamiento de materias primas, materiales y envases. Condiciones y períodos de reaprovisionamiento;

- almacenamiento en proceso. Envejecimiento, cantidades, plazos, requisitos;

- requisitos de transporte y almacenamiento de productos terminados. Cadena frigorifica;
- disponibilidad de servicios de mantenimiento de equipos e instrumentos y de reactivos y otros materiales gastables de los instrumentos. Componentes criticos.

3. Factores humanos (Viña et al, 1994; Viña y Concepción, 1992; Woodson, 1981)

- organización del trabajo, métodos, normas de producción y servicio;

- requisitos del personal. Aptitudes físicas, estado de salud, calificación;

- posibilidades de formación de brigadas, perfil amplio y flexibilidad;

- trabajos en turnos rotativos;

formas de estimulación, salários, emulación;

- otros elementos de atención al hombre. Atención médica, controles biológicos, vacunación, nivel de anticuerpos, tratamiento en caso de embarazo;

personal de mantenimiento. Necesidad de cuidados especiales por exposición al riesgo sin tener la formación adecuada;

- diseño de puestos de trabajo. Flexibilidad. Relación con el régimen de trabalho y descanso;

- formación del o los Comités de Bioseguridad y de Calidad;

- requisitos de iluminación, clima, ambiente sonoro;

- ropas y otros medios de trabajo. Adquisición, control y limpieza; 
- rutinas de alimentación y otras necesidades personales;

- seguridad e higiene ocupacional y ambiental (Englund, 1990);

- control de la exposición. Muestreo del aire. Conteo de la contaminación de fondo (ACGIH, 1989);

- redundancia en los sistemas informativos y de control;

- planes de emergencia (ciclones, inundaciones, explosiones) (Toola, 1992);

- control de la contaminación del medio ambiente. Eliminación de residuos. Incineración y su control. Verificación de las concentraciones máximas admisibles. Efecto de la microlocalización;

- control de la calidad del agua (de entrada y salida);

- reducción de los materiales en inventario, especialmente los peligrosos (inflamables, explosivos, tóxicos, infecciosos, mutágenos, cancerigenos);

- estudios de riesgos y operabilidad (frecuentement se hacen muy tarde);

- sistemas de prevención y extinción de incendios. Caracateristicas de los locales. Sistemas de detección (en los laboratorios de investigación los inventarios son muy pequeños lo que impide que aún en caso de averias las concentraciones alcancen valores peligrosos);

- seguridad de los productos y materiales. Redundancia en almacenes especiales;

- sistema de control de derrames.

\section{Conclusiones}

A partir de la anteriormente planteado, se pueden enunciar las siguientes conclusiones:

- la llamada ingenierización se ha planteado como una solución a los problemas que. pudieran presentarse en el escalado de producciones de la industria Biotecnológica y Farcaméutica;

- existen conceptos y procedimientos que están siendo empleados exitosamente en otras áreas, cuya utilización contribuirá a reducir los problemas que pueden presentearse en el proceso de escalado;

- la ingenieria concurrente ó simultánea es un procedimiento que en particular puede contribuir al aumento de la eficiencia en el proceso de escalado;

- la implementación exitosa de la ingenieria concurrente es posible siguiendo una serie de pasos que difieren del proceso de diseño secuencial usado tradicionalmente.

\section{Referências Bibliográficas}

ACGIH (1989) - Valores limites admisibles para sustancias químicas y agentes físicos en el ambiente de trabajo e indices biológicos de exposición para 19891990, Cronos, Madrid.

Englund, S. M. (1190) - The design and operation of inherently safer chemical plants. Presented at the meeting of the American Institute of Chemical Engineers, San Diego, California. 
Europace System (1992) - Concurrent/ Simultaneous Engineering. Methods, tools and case studies. Course Handouts and tapes (ARTM-12).

Federal Standard 209-D(1990)CleanRooms.

ICIDCA (1992) - La ingenierización de los procesos químicos y bioquímicos, Documento no enumerado, ICIDCA, Mayo, La Habana.

Morita, A. (1992) - A critical moment for japanese management. Economic Eye, Autumn: 4-9.

Patel, B. J. and G. T. R. Humman (1991) Constructing a high-performance, energy-efficient clean room. Microcontamination, Feb. 1991: 29-32.

Rhyne, R. (1991) - If concurrent engineering is the answer, what's the question. En: Proceedings 1991 Frontiers in education conference (IEEE, ASEE), pp. 112116.

Salvendy, G. (1982) - Handbook of industrial engineering, John Wiley \& Sons, New York.

Toola, A. (1992) - Plant level safety analysis. Journal Loss Prev. Process. Ind., 5: (2): 119-124.

Viña, S. (1990) - Guia para la orientación del debate sobre procedimientos de diseño, ISPJAE, La Habana.
12. Viña, S.; E. Concepción y R. Monteiro (1994) - Consideraciones sobre los factores humanos en los poyectos de plantas farmacéuticas y biotecnológicas. Biotecnologia Aplicada (en imprenta).

Viña, S. y E. Concepción (1992) - Algunos aspectos de los factores humanos a tener en cuenta en los poyectos de plantas farmacéuticas. Ponencia presentada en la 1ra. Conferencia Científica Intrernacional de la Universidad de Matanzas, Mayo, Matanzas.

Whalen, J. J. (1991) - Biotechnology regulation. The United State approach. En: Minister of Labour (Ed.): Proceedings Occupational safety and health symposium on biotechnology., pp. 7074, march 21-22, Montreal.

Woodson, W. E. (1981) - Human factors design handbook. Information and quidelines for the design of systems, facilities, equipment and products for human use, McGraw-Hill, New York.

Xerox (1986) Problem-Solving process. User's manual, Xerox corporation, New York.

Ziemke, M. C. and M. S. Spann (1991) Warning: don't be half-hearted in your efforts to employ concurrent engineering. Industrial Engineering, 23: (2): 45-49.

Fecha de terminación: Marzo 1994. 